\title{
Higgs-photon resonances
}

\author{
Bogdan A. Dobrescu, Patrick J. Fox ${ }^{\text {a }}$, John Kearney \\ Theoretical Physics Department, Fermilab, Batavia, IL 60510, USA
}

Received: 2 June 2017 / Accepted: 2 October 2017 / Published online: 24 October 2017

(C) The Author(s) 2017. This article is an open access publication

\begin{abstract}
We study models that produce a Higgs boson plus photon $\left(h^{0} \gamma\right)$ resonance at the LHC. When the resonance is a $Z^{\prime}$ boson, decays to $h^{0} \gamma$ occur at one loop. If the $Z^{\prime}$ boson couples at tree level to quarks, then the $h^{0} \gamma$ branching fraction is typically of order $10^{-5}$ or smaller. Nevertheless, there are models that would allow the observation of $Z^{\prime} \rightarrow$ $h^{0} \gamma$ at $\sqrt{s}=13 \mathrm{TeV}$ with a cross section times branching fraction larger than $1 \mathrm{fb}$ for a $Z^{\prime}$ mass in the $200-450 \mathrm{GeV}$ range, and larger than $0.1 \mathrm{fb}$ for a mass up to $800 \mathrm{GeV}$. The one-loop decay of the $Z^{\prime}$ into lepton pairs competes with $h^{0} \gamma$, even if the $Z^{\prime}$ couplings to leptons vanish at tree level. We also present a model in which a $Z^{\prime}$ boson decays into a Higgs boson and a pair of collimated photons, mimicking an $h^{0} \gamma$ resonance. In this model, the $h^{0} \gamma$ resonance search would be the discovery mode for a $Z^{\prime}$ as heavy as $2 \mathrm{TeV}$. When the resonance is a scalar, although decay to $h^{0} \gamma$ is forbidden by angular momentum conservation, the $h^{0}$ plus collimated photons channel is allowed. We comment on prospects of observing an $h^{0} \gamma$ resonance through different Higgs decays, on constraints from related searches, and on models where $h^{0}$ is replaced by a nonstandard Higgs boson.
\end{abstract}

\section{Contents}

1 Introduction . . . . . . . . . . . . 1

2 Prospects for Higgs-photon resonance searches . . . 2

3 Vector boson decays to $h^{0} \gamma \quad \ldots \ldots$. . . . . . 3

3.1 Dilepton versus $h^{0} \gamma \ldots \ldots \ldots$. . . . . . 4

3.2 Dijet versus $h^{0} \gamma \ldots \ldots \ldots \ldots \ldots$

4 Alternative models with a Higgs-photon resonance . 7

5 Conclusions .................. . . 9

A Appendix: The loop integral . . . . . . . . . . . . . 9

References . . . . . . . . . . . . . . . 10

a e-mail: pjfox@fnal.gov

\section{Introduction}

The ATLAS and CMS experiments at the Large Hadron Collider (LHC) are searching for new particles and interactions in a large number of final states. Among these, a particularly clean class of probes is the resonant production of two Standard Model (SM) particles. A signal of this type would indicate the existence of a new particle that has 2-body decays. Searches for 2-body resonances have covered many combinations of SM particles. Nevertheless, there are some combinations of two SM particles that remain to be searched for at the LHC [1]. Existing resonant searches that involve the Higgs boson, $h^{0}$, and another SM particle in the final state include only $h^{0} W$ and $h^{0} Z$ [2-8].

In this paper we study theoretical and phenomenological constraints on resonances that consist of a Higgs boson and a photon. A particle that can decay into $h^{0} \gamma$ must be a boson. Furthermore, angular momentum conservation prevents that particle from having spin 0 . A simple way to prove that a spin- 0 particle cannot decay into another spin- 0 particle and a photon is to show that the decay amplitude vanishes for any operators that involve these three fields.

Thus, the leading candidate for a $h^{0} \gamma$ resonance is a spin-1 particle, usually known as a $Z^{\prime}$ boson (as it has to be electrically neutral and color singlet). Electromagnetic gauge invariance allows the $Z^{\prime} \rightarrow h^{0} \gamma$ process only through higher-dimensional operators, which arise from loops. Therefore, this partial width is many orders of magnitude smaller than the $Z^{\prime}$ mass. If the $Z^{\prime}$ has sizable couplings to quarks, as required to ensure large $Z^{\prime}$ production at the LHC, then the $B\left(Z^{\prime} \rightarrow h^{0} \gamma\right)$ branching fraction is very small. We give examples of renormalizable $Z^{\prime}$ models, and we compute this $h^{0} \gamma$ branching fraction. We will find that $B\left(Z^{\prime} \rightarrow h^{0} \gamma\right)$ is typically of the order of $10^{-5}$ or smaller. Nevertheless, the observation of the $Z^{\prime}$ in this mode is possible for a range of parameters, due to the small backgrounds.

The signal for a "Higgs-photon" resonance can be much larger than in the case of the loop-induced process if what 
appears to be the photon is in fact a cluster of photons. Consider a heavy boson that decays into a Higgs boson and a spin- 0 particle, $A^{0}$, of GeV-scale mass. If $A^{0}$ subsequently decays into a pair of photons, then the large boost of $A^{0}$ in the lab frame makes the two photons overlap in the electromagnetic calorimeter, leading to a single-photon signature [9-12]. The heavy boson in this case may be a $Z^{\prime}$, or even a spin- 0 particle given that its decay into $h^{0} A^{0}$ conserves angular momentum. Eventually, with more detailed studies and larger data sets, the collimated photons (collectively labeled " $\gamma$ ") may be distinguished from a single photon.

In Sect. 2 we discuss phenomenological issues associated with possible Higgs-photon resonance searches, and estimate the current cross-section limits based on related experimental results. The $Z^{\prime}$ models and their predictions for resonant searches at the LHC are presented in Sect. 3. The collimated-photon scenario is discussed in Sect. 4. There, we also describe a renormalizable model that leads to $Z^{\prime} \rightarrow$ $h^{0} A^{0} \rightarrow h^{0}+$ " $\gamma$ ". Section 5 includes our conclusions as well as some comments on $h^{\prime} \gamma$ resonances, where $h^{\prime}$ is a new Higgs-like boson.

\section{Prospects for Higgs-photon resonance searches}

There are currently no published searches for $h^{0} \gamma$ resonances. Yet, such searches could provide an interesting test of physics beyond the SM. In this section we discuss the prospects for such a search at the LHC, with particular focus on the current experimental reach that could be achieved with minimal extension of pre-existing searches in related channels.

We concentrate on the final state in which the Higgs boson decays into bottom quarks. We will denote the decaying particle by $Z^{\prime}$ in this section, as for a new vector boson, but our results are more generally applicable (the case of a spin0 resonance is briefly discussed in Sect. 4). First, consider a light resonance, $M_{Z^{\prime}} \lesssim 700 \mathrm{GeV}$, so that the final state is $b \bar{b} \gamma$ with resolved $b$ jets. The dominant SM background is non-resonant $b \bar{b} \gamma$ production and, to a lesser extent, $j j \gamma$ with mis-tags.

In estimating the background rate we require that $110 \mathrm{GeV}<m_{b \bar{b}, j j}<135 \mathrm{GeV}$, that $p_{T}>50 \mathrm{GeV}$ for jets, $b$ jets and photons, and we assume a $b$-tagging efficiency of $70 \%$ with a $b$ jet fake rate of $1 \%$. After applying these cuts and efficiencies we find, based on simulation with MadGraph [13], that the $b \bar{b} \gamma(j j \gamma)$ background cross section at the $13 \mathrm{TeV}$ LHC is $\sim 250 \mathrm{fb}(5 \mathrm{fb})$. In both cases the distribution of $m_{b \bar{b} \gamma, j j \gamma}$ is peaked at low invariant mass. The large background will make a search in this regime challenging. However, various kinematic features of the signal, including the resonant peak in $m_{b \bar{b} \gamma}$, can be used to differentiate the signal from the background [14]. Since the search is for a resonance, we expect the discovery reach is limited by statistics. Ignoring systematic uncertainties a signal cross sections even below $\sim 1 \mathrm{fb}$ can be observed, with $3000 \mathrm{fb}^{-1}$ of data. Moreover, as we discuss in the context of specific models below, low-mass $h^{0} \gamma$ resonances may offer the best prospects for observation due to the larger rates. This makes the development of a dedicated search strategy for such resonances particularly important.

For a heavier $Z^{\prime}$ boson, $M_{Z^{\prime}} \gtrsim 700 \mathrm{GeV}$, the Higgs boson will be boosted and the two $b$ quarks will be contained within a jet of size $\Delta R \sim 2 M_{h} / p_{T, h} \lesssim 0.7$. Thus, the observed final state is a photon and a wide jet with substructure. As the final state $(\gamma j)$ is similar to that considered in searches for excited quarks, we can reinterpret the results of these searches to estimate the current and future reach of a dedicated $h^{0} \gamma$ search in this regime.

The dominant backgrounds for the excited quark search are continuum $\gamma j$ as well as QCD with jet misidentification [15-18]. However, these backgrounds can be efficiently suppressed in the case of $h^{0} \gamma$ by applying a Higgs tagger to the jet, as for the $W h^{0}$ resonance search [5]. The Higgs tagger requires the jet mass to lie close to the Higgs mass, and it requires the presence of a heavy flavor tag in the jet, and is $\simeq 80 \%$ efficient while having $\mathrm{a} \simeq 10 \%$ jet misidentification rate [5]. Estimating the effect of applying the Higgs tagger directly to the existing excited quark search is complicated by the fact that the tagger is based on Cambridge-Aachen jets with $\Delta R=0.8$ while jets in the excited quark search are clustered according to the anti- $k_{T}$ algorithm with $\Delta R=0.5$. Increasing the jet radius leads to more background jets passing the cut $p_{T, j}>170 \mathrm{GeV}$ applied in [15]. We estimate this increase to be around $10 \%$, by simulating the dominant direct background $p p \rightarrow \gamma j$ in MadGraph [13], with showering carried out subsequently in PYTHIA 6.4 [20] and detector simulation using DELPHES 3.3.0 [21]. Jets are clustered both ways using FastJet [22], and the proportion of events passing the experimental cuts is used to estimate the impact of changing jet algorithms. Overall, the effect of increasing the jet radius and applying the Higgs tagger is expected to reduce the background by a factor of approximately 10.

The CMS excited quark search [15] does not present the acceptance times efficiency for the $q^{*} \rightarrow q \gamma$ signal to pass the analysis, so we must estimate this quantity. For a given $q^{*}$ resonance mass $M$ we estimate the number of expected background events with $\left|m_{\gamma j}-M\right|<0.12 M$, which corresponds to a $3 \sigma$ window for the CMS resolution, using the functional form for the background provided in [15]. By comparing our estimated limits, based on these background estimates, with those published by the collaboration, we determine an approximately mass-independent acceptance times efficiency, $\mathcal{A} \epsilon \approx 0.5$, for $q^{*}$ signal events. This estimate is consistent with the approximate efficiency given in $[17,18]$, 


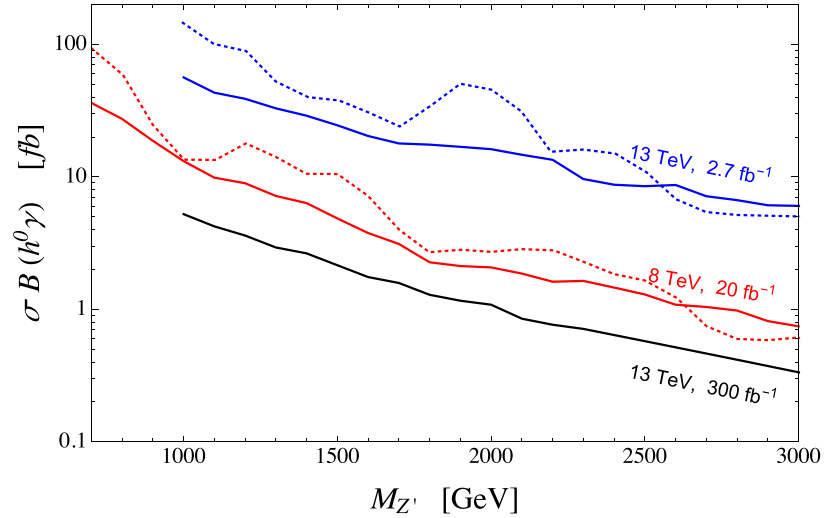

Fig. 1 Estimated limits on the cross section for an $h^{0} \gamma$ resonance obtained by recasting the $p p \rightarrow q^{*} \rightarrow \gamma q$ search results: current limits at $\sqrt{s}=13 \mathrm{TeV}$ and $8 \mathrm{TeV}$ (solid blue and red lines, respectively), and projected limit at $13 \mathrm{TeV}$ with $\mathcal{L}=300 \mathrm{fb}^{-1}$ (solid black line). For comparison, dotted lines represent the current CMS $[15,16]$ limits on the cross section times branching fraction for an excited quark decaying $q^{*} \rightarrow \gamma q$; the improvement by a factor of approximately 1.5 over much of the mass range is the result of applying the Higgs tagger

and agrees well with Fig. 1 of the very recent work in Ref. [19].

Assuming that $\mathcal{A} \in$ for our signal is the same as that derived for an excited quark we estimate the reach of an excited quark search applied to an $h^{0} \gamma$ resonance. Due to changes in jet size and mistagging rates the background is rescaled by $\simeq 0.11$, and the signal rate is given by $0.8 \mathcal{A} \in \sigma B\left(Z^{\prime} \rightarrow\right.$ $\left.h^{0} \gamma\right) B\left(h^{0} \rightarrow b \bar{b}\right)$. Using excited quark searches both at 8 $\mathrm{TeV}$ with $\mathcal{L}=20 \mathrm{fb}^{-1}[15]$ and $13 \mathrm{TeV}$ with $\mathcal{L}=2.7 \mathrm{fb}^{-1}$ [16], we project the current limits on an $h^{0} \gamma$ resonance that would be obtained were a search similar to that described here implemented by the CMS collaboration. We also use these limits to project the reach that could be obtained at 13 $\mathrm{TeV}$ with $\mathcal{L}=300 \mathrm{fb}^{-1}$. These limits are shown in Fig. 1. Thus, we estimate that the cross-section sensitivity to an $h^{0} \gamma$ resonance in the mass range $1-3 \mathrm{TeV}$ at the $8 \mathrm{TeV} \mathrm{LHC}$ ranges from 10 to $1 \mathrm{fb}$, and at the $13 \mathrm{TeV}$ LHC with $300 \mathrm{fb}^{-1}$ of data will be ranging from 5 to $0.5 \mathrm{fb}$. Comparing the 8 and $13 \mathrm{TeV}$ reach, the background increases by a factor of about 3 , while the increase in the signal cross section depends on the initial state, which is model dependent.

Additional Higgs decays can be used to search for $h^{0} \gamma$ resonances. For example, a search in the a photon-plus-lepton final state would be sensitive to $h^{0} \rightarrow W W^{*} \rightarrow \ell v j j$ and $h^{0} \rightarrow \tau^{+} \tau^{-}$. Also, the production of an $h^{0} \gamma$ resonance followed by the Higgs boson decays into photon pairs would lead to a spectacular $3 \gamma$ peak, which would allow a precise determination of the resonance mass. There is an ATLAS search for a $3 \gamma$ resonance [23], but it is not sensitive enough to be applied to $h^{0} \gamma$ resonances because the Higgs branching fraction into photons is too small.
Other $Z^{\prime}$ decay channels may be important. The relative branching fractions and the search sensitivities of various channels will determine whether $h^{0} \gamma$ represents a potential discovery channel for the new resonance or a precision probe of a resonance discovered in another channel. The possible $Z^{\prime}$ decays are model dependent, but certain other channels may be particularly relevant. For instance, as the $Z^{\prime}$ must be produced in hadron collisions, it is likely to have a sizable branching fraction to jets. Meanwhile, for a vector resonance, the $Z^{\prime}$ may also be able to decay to lepton pairs, as will be discussed in the next section. Comparing the estimated $8 \mathrm{TeV}$ sensitivity to $h^{0} \gamma$ with those to dijet [24] and dilepton [25] resonances, we find that the dijet channel is 10-100 times less sensitive in the high mass regime, while the dilepton channel is approximately 10-30 times more sensitive. Below, we will discuss the impact of these other channels, and their importance for interpreting the results of an $h^{0} \gamma$ search in specific models.

\section{Vector boson decays to $h^{0} \gamma$}

A new vector boson $Z^{\prime}$ can decay to $h^{0} \gamma$ via an operator of the form

$\frac{c_{\gamma} e v}{(4 \pi)^{2} m_{0}^{2}} h Z_{\mu \nu}^{\prime} F^{\mu \nu}$

where $Z_{\mu \nu}^{\prime}=\partial_{\mu} Z_{\nu}^{\prime}-\partial_{\nu} Z_{\mu}^{\prime}$, and $F_{\mu \nu}$ is the electromagnetic field strength; $m_{0}$ is the mass of some particle running in the loop, $e \approx 0.3$ is the electromagnetic gauge coupling, $v \approx 246 \mathrm{GeV}$ is the weak scale. The factor of $(4 \pi)^{2}$ in the denominator is associated with the loop integral, so that the model-dependent dimensionless parameter $c_{\gamma}$ is typically of order one or smaller.

An additional operator, $h \widetilde{Z}_{\mu \nu}^{\prime} F^{\mu \nu}$, can also contribute to $Z^{\prime} \rightarrow h^{0} \gamma$. However, the coefficient of that operator vanishes in the limit of CP conservation, and we will ignore it here. $U(1)_{\mathrm{em}}$ gauge invariance ensures any additional operators (including higher dimension operators) contributing to onshell decay $Z^{\prime} \rightarrow h^{0} \gamma$ can be related to operator (3.1) using the equations of motion. A straightforward way to see this is to consider the matrix element for the decay. The Ward identity requires $p_{\mu}(\gamma) \epsilon_{v}\left(Z^{\prime}\right) \mathcal{M}^{\mu v}=0$, where the full matrix element is $\mathcal{M}=\epsilon_{\mu}^{*}(\gamma) \epsilon_{\nu}\left(Z^{\prime}\right) \mathcal{M}^{\mu \nu}$ with $\epsilon$ the polarization of the $Z^{\prime}$ boson or photon. So, writing $\mathcal{M}^{\mu \nu}$ in terms of momenta, it must be the case that

$\mathcal{M}^{\mu \nu} \propto p^{\mu}\left(Z^{\prime}\right) p^{\nu}(\gamma)-p\left(Z^{\prime}\right) \cdot p(\gamma) g^{\mu \nu}$,

i.e., we have the tensor structure that arises from operator (3.1). 


\subsection{Dilepton versus $h^{0} \gamma$}

The form of the operator responsible for the decay $Z^{\prime} \rightarrow h^{0} \gamma$ immediately indicates that a vector resonance decaying to $h^{0} \gamma$ is likely to exhibit decays to lepton pairs. Specifically, as can be seen by replacing $h$ with its VEV, whatever physics gives rise to operator (3.1) should also generate a kinetic mixing of $Z^{\prime}$ with the photon. A kinetic mixing of the $Z^{\prime}$ and $Z$ bosons is also likely to be present. The mixing terms in the Lagrangian can be written as

$\frac{e v^{2}}{2(4 \pi)^{2} m_{0}^{2}} Z_{\mu \nu}^{\prime}\left(\tilde{c}_{\gamma} F^{\mu \nu}+\frac{\tilde{c}_{Z}}{s_{W} c_{W}} Z^{\mu \nu}\right)$.

The coefficient $\tilde{c}_{\gamma}$ is different from $c_{\gamma}$ in order to take into account contributions to the kinetic mixing which are not related to electroweak symmetry breaking. The dimensionless parameter $\tilde{c}_{Z}$ is also model dependent; $g=e / s_{W}$ is the $S U(2)_{W}$ coupling, and $c_{W} \equiv \cos \theta_{W}, s_{W} \equiv \sin \theta_{W}$, where $\theta_{W}$ is the weak mixing angle.

The kinetic mixing would generically allow $Z^{\prime}$ to decay to additional states, notably lepton pairs. The impressive sensitivity exhibited by dilepton resonance searches at the LHC means that if the dilepton and $h^{0} \gamma$ decay rates are comparable, $\Gamma\left(Z^{\prime} \rightarrow \ell^{+} \ell^{-}\right) \gtrsim \Gamma\left(Z^{\prime} \rightarrow h^{0} \gamma\right)$, the new resonance may be first observed in dileptons. In this case, an $h^{0} \gamma$ search would be an important part of fully characterizing the $Z^{\prime}$, and as a probe of the physics responsible for generating the kinetic mixing and loop-level decay $Z^{\prime} \rightarrow h^{0} \gamma$. The $h^{0} \gamma$ search would be facilitated by knowing $M_{Z^{\prime}}$ from the dilepton search. Alternatively, if the dilepton decay rate is subdominant, $h^{0} \gamma$ may represent a viable discovery channel.

To elucidate which situation may be most likely in different regions of parameter space, let us estimate the relative rates of the $Z^{\prime} \rightarrow h^{0} \gamma$ and $Z^{\prime} \rightarrow \ell^{+} \ell^{-}$channels. The partial widths are

$\Gamma\left(Z^{\prime} \rightarrow h^{0} \gamma\right)=\frac{c_{\gamma}^{2} \alpha v^{2}}{1536 \pi^{4} m_{0}^{4}} M_{Z^{\prime}}^{3}\left(1-\frac{M_{h}^{2}}{M_{Z^{\prime}}^{2}}\right)^{3}$,

and, to leading order in the parameters $\tilde{c}_{\gamma}, \tilde{c}_{Z}$ (i.e., assuming the kinetic mixing is small)

$$
\begin{aligned}
& \Gamma\left(Z^{\prime} \rightarrow e^{+} e^{-}\right)=\Gamma\left(Z^{\prime} \rightarrow \mu^{+} \mu^{-}\right) \\
& \quad=\left(\tilde{c}_{\gamma}^{2}+\tilde{c}_{\gamma} \tilde{c}_{Z} a_{W}+\tilde{c}_{Z}^{2} b_{W}\right) \frac{\alpha^{2} v^{4}}{192 \pi^{3} m_{0}^{4}} M_{Z^{\prime}},
\end{aligned}
$$

where we defined

$$
\begin{aligned}
& a_{W}=\frac{1-4 s_{W}^{2}}{2 c_{W}^{2} s_{W}^{2}}+O\left(M_{Z}^{2} / M_{Z^{\prime}}^{2}\right) \approx 0.21, \\
& b_{W}=\frac{1-4 s_{W}^{2}+8 s_{W}^{4}}{8 c_{W}^{4} s_{W}^{4}}+O\left(M_{Z}^{2} / M_{Z^{\prime}}^{2}\right) \approx 2.0 .
\end{aligned}
$$

Thus, the ratio of branching fractions to $h^{0} \gamma$ and lepton pairs (i.e., the sum over $e^{+} e^{-}$and $\mu^{+} \mu^{-}$) can be parametrized as follows:

$$
\frac{B\left(Z^{\prime} \rightarrow h^{0} \gamma\right)}{B\left(Z^{\prime} \rightarrow \ell^{+} \ell^{-}\right)}=r_{h \gamma}\left(\frac{M_{Z^{\prime}}}{1 \mathrm{TeV}}\right)^{2}\left(1-\frac{M_{h}^{2}}{M_{Z^{\prime}}^{2}}\right)^{3},
$$

where $r_{h \gamma}$ is a dimensionless parameter that depends on the coefficients $c_{\gamma}, \tilde{c}_{\gamma}$ and $\tilde{c}_{Z}$.

Consider the case where the only $S U(2)_{W} \times U(1)_{Y^{-}}$ invariant operator responsible for $Z^{\prime} \rightarrow h^{0} \gamma$ and $Z^{\prime} \rightarrow$ $\ell^{+} \ell^{-}$is

$\frac{C_{H} e}{(4 \pi)^{2} m_{0}^{2} c_{W}} H^{\dagger} H Z_{\mu \nu}^{\prime} B^{\mu \nu}$,

where $H$ is the SM Higgs doublet, and $B^{\mu v}$ is the hypercharge field strength. The coefficients then satisfy $c_{\gamma}=\tilde{c}_{\gamma}=C_{H}$ and $\tilde{c}_{Z}=-C_{H} s_{W}^{2}$, so that $r_{h \gamma} \approx 40$. From Eq. (3.7) it then follows that $B\left(Z^{\prime} \rightarrow h^{0} \gamma\right)>B\left(Z^{\prime} \rightarrow \ell^{+} \ell^{-}\right)$for $M_{Z^{\prime}} \gtrsim 247 \mathrm{GeV}$. The above operator also induces a decay to $h^{0} Z$, which we do not discuss here as it is a less sensitive search mode than $h^{0} \gamma$ due to the small leptonic $Z$ branching fraction.

Another example of an operator that induces $Z^{\prime}$ decays into $h^{0} \gamma$ and $\ell^{+} \ell^{-}$is

$$
-\frac{C_{H}^{\prime} g}{(4 \pi)^{2} m_{0}^{2}} H^{\dagger} \sigma^{a} H Z_{\mu \nu}^{\prime} W^{a \mu \nu} \text {. }
$$

If this single operator contributes to these decays, then $c_{\gamma}=$ $\tilde{c}_{\gamma}=C_{H}^{\prime}$ and $\tilde{c}_{Z}=C_{H}^{\prime} c_{W}^{2}$, which implies $r_{h \gamma} \approx 18$, and the $h^{0} \gamma$ branching fraction is larger than the dilepton one for $M_{Z^{\prime}} \gtrsim 309 \mathrm{GeV}$.

The coefficients of the kinetic terms, $\tilde{c}_{\gamma}$ and $\tilde{c}_{Z}$, can also receive contributions which are independent of electroweak symmetry breaking. We assume that the $Z^{\prime}$ boson is associated with a $U(1)$ gauge symmetry. A tree-level dimension4 operator $Z_{\mu \nu}^{\prime} B^{\mu \nu}$ may be eliminated by embedding one of the Abelian gauge groups in a larger group at some high scale. If there are fields that carry both hypercharge and $U(1)$ charges though, this kinetic mixing may be generated at one loop, but with a model-dependent coefficient. In the particular case where all the fields charged under both groups have the same mass and the product of hypercharge and $U(1)$ charge summed over all fields is zero, the one-loop contribution to $Z_{\mu \nu}^{\prime} B^{\mu \nu}$ vanishes. Thus, it is possible that the dominant contribution to $Z^{\prime} \rightarrow \ell^{+} \ell^{-}$arises from the operators (3.8) or (3.9). This might not be the case if the SM quarks carry the new $U(1)$ charges, as explained towards the end of the next subsection.

\subsection{Dijet versus $h^{0} \gamma$}

Kinetic mixing also generates a decay to dijets. The large background to dijet resonance searches ensures that, for 
$\Gamma\left(Z^{\prime} \rightarrow j j\right)$ comparable to $\Gamma\left(Z^{\prime} \rightarrow h^{0} \gamma\right)$ or $\Gamma\left(Z^{\prime} \rightarrow\right.$ $\left.\ell^{+} \ell^{-}\right), h^{0} \gamma$ and dilepton searches would represent more promising discovery channels for the new state. However, a model with an appreciable $Z^{\prime}$ production (and hence $h^{0} \gamma$ ) rate typically implies a significant decay rate to dijets, beyond the small rate induced via kinetic mixing. In particular, the $Z^{\prime}$ production at the LHC is large provided there are tree-level couplings of the first-generation quarks to the $Z^{\prime}$. In that case the width of the $Z^{\prime}$ decay mode into a pair of jets is several orders of magnitude larger than the width of $Z^{\prime} \rightarrow h^{0} \gamma$. Nevertheless, due to the large dijet background, $h^{0} \gamma$ could be the discovery mode in some cases. In this subsection, we consider a model based on a leptophobic $Z^{\prime}$, and demonstrate that an interesting rate for $p p \rightarrow Z^{\prime} \rightarrow h^{0} \gamma$ can still be achieved consistent with constraints from dijet resonance searches, particularly at lower $M_{Z^{\prime}}$.

Let us compute precisely the one-loop $h^{0} \gamma$ width, $\Gamma\left(Z^{\prime} \rightarrow\right.$ $h^{0} \gamma$ ), in the case where a fermion $F$ of mass $m_{F}$ and electric charge $Q$ has a Yukawa coupling $\left(y_{F} / \sqrt{2}\right) h^{0} \bar{F} F$ to the Higgs boson, and couples vectorially to the $Z^{\prime}$ :

$\frac{g_{z}}{2} z_{F} Z_{\mu}^{\prime}\left(\bar{F}_{L} \gamma^{\mu} F_{L}+\bar{F}_{R} \gamma^{\mu} F_{R}\right)$,

where $g_{z}$ is the gauge coupling, and $z_{F}$ is the charge of the fermion under the new gauge group. There are two diagrams, shown in Fig. 2, which contribute to $Z^{\prime} \rightarrow h^{0} \gamma$. Defining the mass ratios

$r_{h}=\frac{M_{h}}{M_{Z^{\prime}}}, \quad r_{F}=\frac{m_{F}}{M_{Z^{\prime}}}$,

we find that the one-loop width induced by fermion $F$ is

$\Gamma\left(Z^{\prime} \rightarrow h^{0} \gamma\right)=\frac{\left(N_{c} Q_{F}\right)^{2} \alpha}{384 \pi^{4}}\left(y_{F} g_{z} z_{F}\right)^{2} M_{Z^{\prime}} f\left(r_{h}^{2}, r_{F}^{2}\right)$,

where $N_{c}$ is the number of colors of the fermion. The dimensionless function $f$, computed in the appendix, includes the loop integral and the phase space.

In the case where $F$ is the top quark, $N_{c}=3, Q_{F}=2 / 3$, and $y_{F}$ is the SM top Yukawa coupling: $y_{t}=\sqrt{2} m_{t} / v \approx 1$. Note that the contributions from lighter quarks are suppressed by their mass squared. Assuming flavor-universal vector couplings of $Z^{\prime}$ to the SM quarks, and that no other particles contribute to the loop process, the ratio of the $h^{0} \gamma$ and dijet widths is

$$
\frac{\Gamma\left(Z^{\prime} \rightarrow h^{0} \gamma\right)}{\sum_{q} \Gamma\left(Z^{\prime} \rightarrow q \bar{q}\right)} \simeq \frac{\alpha y_{t}^{2} f\left(r_{h}^{2}, r_{t}^{2}\right)}{6 \pi^{3}\left(5+\left(1-r_{t}^{2}\right) \sqrt{1-4 r_{t}^{2}}\right)},
$$

where $r_{t} \equiv m_{t} / M_{Z^{\prime}}$. If the $Z^{\prime}$ does not interact with new particles coupled to the Higgs doublet, then the maximum value of the $Z^{\prime} \rightarrow h^{0} \gamma$ branching fraction occurs for $M_{Z^{\prime}}=$ $2 m_{t}: B\left(Z^{\prime} \rightarrow h^{0} \gamma\right)^{\max }=2.3 \times 10^{-5}$. The $h^{0} \gamma$ branching fraction is plotted as a function of $M_{Z^{\prime}}$ in the left panel of Fig. 3.

A $Z^{\prime}$ boson with flavor-universal couplings to all SM quarks arises in the presence of an extension of the SM gauge group by a $U(1)_{B}$ symmetry, with all quarks carrying the same charge (by convention $z_{F}=1 / 3$ while the gauge coupling $g_{z}$ is a free parameter). The cancellation of the gauge anomalies involving $U(1)_{B}$ requires new fermions (called anomalons), which must be chiral with respect to $U(1)_{B}$, and are constrained to be vectorlike with respect to the SM gauge group. Specific sets of anomalons were introduced in Refs. [26-32]. The couplings of the anomalons to the Higgs doublet are model dependent. In the limit where these vanish, the anomalons do not contribute to the $Z^{\prime} \rightarrow h^{0} \gamma$ width.

The $Z^{\prime}$ production cross section is proportional to $g_{z}^{2}$. We have computed the inclusive leading-order production cross section of the $Z^{\prime}, \sigma\left(p p \rightarrow Z^{\prime}+X\right)$, at the $13 \mathrm{TeV}$ LHC using MadGraph [13], with model files generated by FeynRules [33]. In the right panel of Fig. 3 we plot the cross section times the branching fraction, $\sigma\left(p p \rightarrow Z^{\prime}+X\right) B\left(Z^{\prime} \rightarrow\right.$ $h^{0} \gamma$ ), as a function of $M_{Z^{\prime}}$, for $Z^{\prime}$ gauge coupling $g_{z}=0.3$ or 0.1 . We also show the upper limit imposed by various dijet resonance searches [34-40], which constrain the gauge coupling [30] in this leptophobic $Z^{\prime}$ model. Even though the loop generated decay has a small branching fraction, the $h^{0} \gamma$ resonance searches can still compete with the dijet resonance searches. The $\sigma\left(p p \rightarrow Z^{\prime} \rightarrow h^{0} \gamma\right)$ cross section at $\sqrt{s}=13$ $\mathrm{TeV}$ can be larger than $1 \mathrm{fb}$ for $M_{Z^{\prime}}<450 \mathrm{GeV}$, while values larger than $0.1 \mathrm{fb}$ are allowed for $M_{Z^{\prime}}<550 \mathrm{GeV}$.

If particles beyond the SM carry electric and $U(1)_{B}$ charges, and also couple to the Higgs boson, then their oneloop contributions interfere with the SM quark loops and may enhance or decrease the $Z^{\prime} \rightarrow h^{0} \gamma$ branching fraction. Let us consider a simple extension of the SM with two vectorlike leptons carrying $U(1)_{B}$ charge -1 : one is a weak-doublet of hypercharge $-1 / 2$ (same as the SM lepton doublets) labeled $\psi_{D}=\left(\psi_{D}^{v}, \psi_{D}^{e}\right)$, and the other one is a weak-singlet of hypercharge -1 labeled $\psi_{S}$. These have gauge-invariant masses as well as a Yukawa coupling to the SM Higgs doublet,

$$
-m_{D} \bar{\psi}_{D} \psi_{D}-m_{S} \bar{\psi}_{S} \psi_{S}-y_{\psi}\left(\bar{\psi}_{D} \psi_{S} H+\text { H.c. }\right) \text {. }
$$

The two electrically charged fermions mix, giving rise to the following mass-eigenstates:

$$
\left(\begin{array}{l}
\psi_{1} \\
\psi_{2}
\end{array}\right)=\left(\begin{array}{cc}
\cos \theta & \sin \theta \\
-\sin \theta & \cos \theta
\end{array}\right)\left(\begin{array}{l}
\psi_{D}^{e} \\
\psi_{S}
\end{array}\right),
$$

where the mixing angle satisfies

$$
\tan 2 \theta=\frac{\sqrt{2} y_{\psi} v}{m_{D}-m_{S}} .
$$



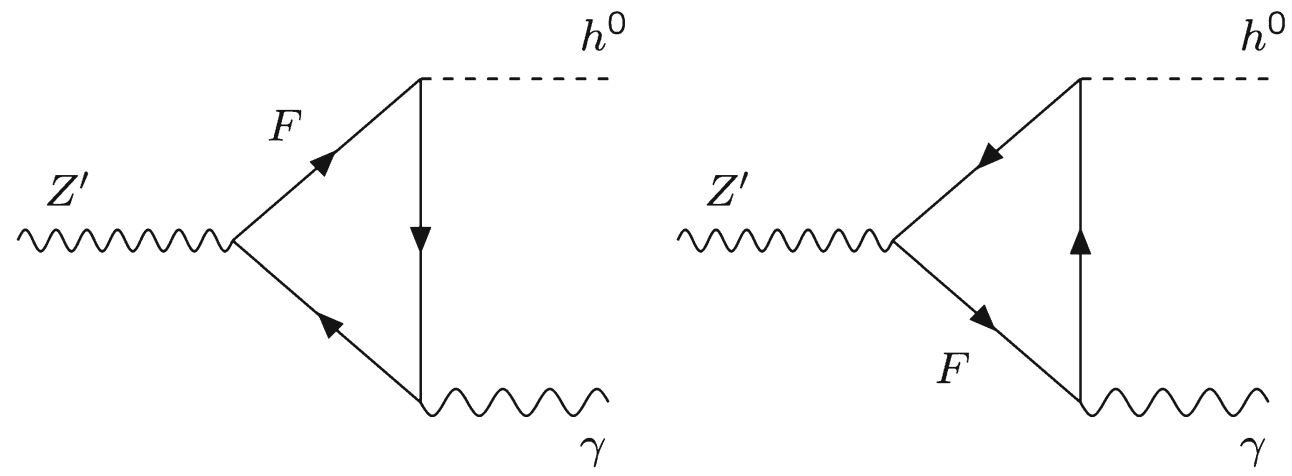

Fig. 2 One-loop contributions of a fermion $F$ to the $Z^{\prime} \rightarrow h^{0} \gamma$ amplitude

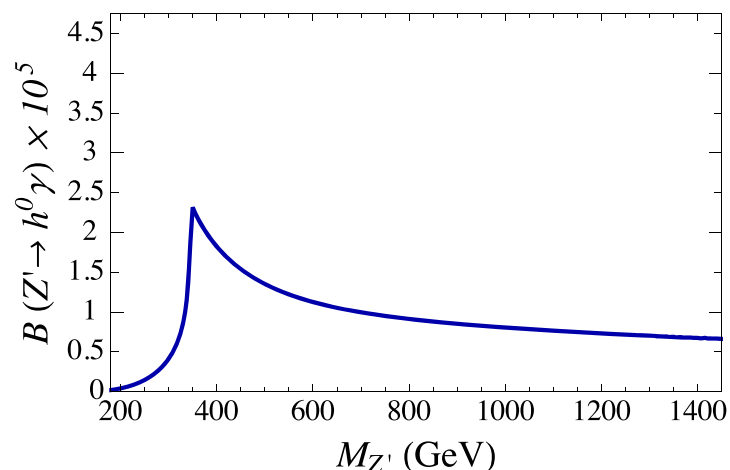

Fig. 3 Left panel The $Z^{\prime} \rightarrow h^{0} \gamma$ branching fraction as a function of the $Z^{\prime}$ mass, when the $Z^{\prime}$ has flavor-universal vector couplings to the SM quarks, and there are no new particles running in the loop. Right panel Leading-order $p p \rightarrow Z^{\prime} \rightarrow h^{0} \gamma$ cross section at $\sqrt{s}=13 \mathrm{TeV}$

Labeling the mass of the lightest physical state by $m_{\psi}$, the mass of the other charged vectorlike lepton is

$m_{\psi^{\prime}}=m_{\psi}+\frac{\sqrt{2} y_{\psi} v}{\sin 2 \theta}$.

The $Z^{\prime} \rightarrow h^{0} \gamma$ width, given in Eq. (3.13) when the only large contribution is from the top quark, is modified in this case by a factor of

$\left|1+\frac{3 y_{\psi}}{2 y_{t}} \sin 2 \theta \frac{I\left(r_{h}, r_{\psi}\right)+I\left(r_{h}, r_{\psi^{\prime}}\right)}{I\left(r_{h}, r_{t}\right)}\right|^{2}$,

where $r_{\psi}=m_{\psi} / M_{Z^{\prime}}, r_{\psi^{\prime}}=m_{\psi^{\prime}} / M_{Z^{\prime}}$, and $I$ is the loop integral given in Eq. (A.2). The $Z^{\prime} \rightarrow h^{0} \gamma$ branching fraction is shown in Fig. 4 for $\sin \theta=0.3, y_{\psi}=1$ and $m_{\psi}=300$ $\mathrm{GeV}$ or $500 \mathrm{GeV}$. The $p p \rightarrow Z^{\prime} \rightarrow h^{0} \gamma$ cross section at the $13 \mathrm{TeV}$ LHC is shown in the right panel of Fig. 4 for two values of the gauge coupling.

The limits on vectorlike lepton masses from collider searches are model dependent, and they are rather loose for decays into $W v, \tau Z$, or $\tau h^{0}$ [41]. When the vectorlike leptons are heavier than the $Z^{\prime}$ boson, they can decay into a $Z^{\prime}$

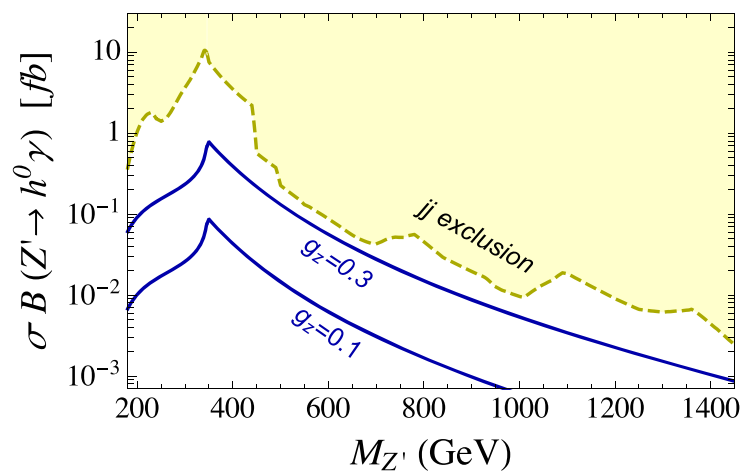

for two values of the gauge couplings $g_{z}$, when all the SM quarks have charge $z_{F}=1 / 3$ under the new gauge group. The shaded region is ruled out by current dijet resonance limits

and a SM lepton, but again the LHC sensitivity is reduced when the lepton is a $\tau$. The constraints on the Higgs Yukawa coupling of the vectorlike leptons from the measurements of $h^{0} \rightarrow \gamma \gamma$ are also loose.

Let us now comment on the expected size of the kinetic mixing and associated dilepton decay rate in this model. To ensure $Z^{\prime} \rightarrow \ell^{+} \ell^{-}$does not dominate over $Z^{\prime} \rightarrow h^{0} \gamma$, the tree-level kinetic mixing between the $U(1)_{Y}$ and $U(1)_{B}$ gauge bosons is assumed to vanish at a scale $\Lambda$, of order 10 $\mathrm{TeV}$ or higher. As mentioned earlier, this can be enforced, for example, by embedding one of the $U(1)$ gauge groups in a non-Abelian group at scale $\Lambda$. Furthermore, for the sets of anomalons given in $[31,32]$, which include only 8 Weyl fermions, the sum over anomalons of the product of hypercharge and $U(1)_{B}$ charge cancels the analogous sum over SM quarks. As the field content of this model satisfies $\operatorname{Tr}(Y B)=0$, the dimension-4 kinetic mixing is not induced at one loop above the mass of the anomalons, $m_{a}$. Below this mass, however, the $Z_{\mu \nu}^{\prime} B^{\mu \nu}$ kinetic mixing parameter will run. Since the contribution from the anomalons cancels against the contribution from the SM fermions, the ratio of the kinetic mixing to $c_{\gamma}$ is proportional to $\log \left(m_{a} / M_{Z^{\prime}}\right)$. Thus, 


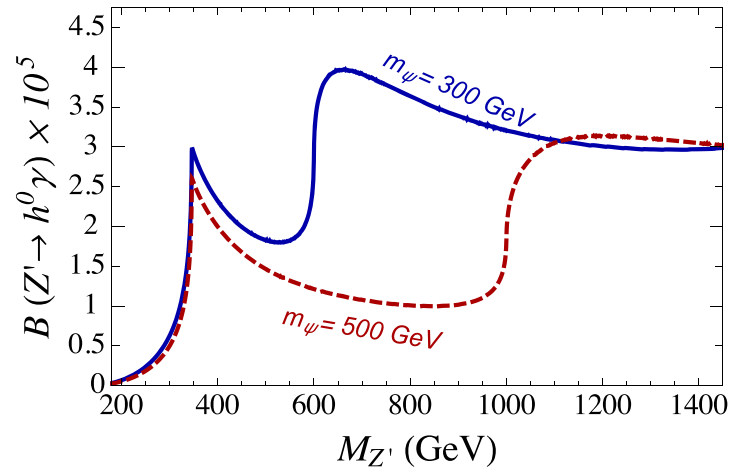

Fig. 4 Left panel The $Z^{\prime} \rightarrow h^{0} \gamma$ branching fraction for flavoruniversal vector couplings of $Z^{\prime}$ to the SM quarks with $z_{F}=1 / 3$, when two vectorlike leptons with $z_{\psi}=-1$ are running in the loop. The mass of lightest vectorlike lepton is $m_{\psi}=300 \mathrm{GeV}$ (blue solid line) or $500 \mathrm{GeV}$ (dashed red line); the mixing angle and the Higgs

if the anomalon and $Z^{\prime}$ masses are comparable, then $\tilde{c}_{\gamma}$ and $\tilde{c}_{Z}$ are of the same order as $c_{\gamma}$. As a result, $h^{0} \gamma$ and dilepton resonance searches represent complementary probes of the model, and either may serve as a possible discovery channel.

While the couplings of anomalons to the SM Higgs boson are model dependent and may even vanish, the anomalon couplings to the scalar field, $\phi$, breaking $U(1)_{B}$, are necessary to generate their masses. Thus, anomalon loops induce an additional dimension- 6 operator:

$\frac{C_{\phi} e}{(4 \pi)^{2} m_{0}^{2} c_{W}} \phi^{\dagger} \phi Z_{\mu \nu}^{\prime} B^{\mu \nu}$.

This contributes to the kinetic mixing when $\phi$ is replaced by its VEV, but also leads to other notable experimental signatures. The SM Higgs boson and the $\mathrm{CP}$-even component of $\phi$ can mix through the Higgs portal $|\phi|^{2}|H|^{2}$. The physical states are the observed Higgs-like particle $(h)$ of mass $M_{h} \approx 125 \mathrm{GeV}$, and a second scalar $h^{\prime}$ of mass $M_{h^{\prime}}$. The mixing leads to $h^{\prime}$ decay to pairs of SM fermions and vector bosons. For $M_{h^{\prime}}<M_{Z^{\prime}}$, the decay $Z^{\prime} \rightarrow h^{\prime} \gamma$ with $h^{\prime}$ decaying as a Higgs-like particle could also be searched for. For $10 \mathrm{GeV} \lesssim M_{h^{\prime}} \lesssim 160 \mathrm{GeV}$ the search channel would be $b \bar{b} \gamma$ with the $b \bar{b}$ resonance no longer at $M_{h}$. For higher $M_{h^{\prime}}$ masses a combination of $W^{+} W^{-} \gamma, Z Z \gamma$, and $t \bar{t} \gamma$, again with subresonances at the $h^{\prime}$ mass, would be the dominant search channels.

\section{Alternative models with a Higgs-photon resonance}

In the preceding section, we discussed models that include a spin-1 particle that could give rise to an $h^{0} \gamma$ signal at the LHC. While this motivates the implementation of a dedicated $h^{0} \gamma$ resonance search, $Z^{\prime} \rightarrow h^{0} \gamma$ seems to be most interesting for $M_{Z^{\prime}} \lesssim 800 \mathrm{GeV}$, where the weaker dijet limits still

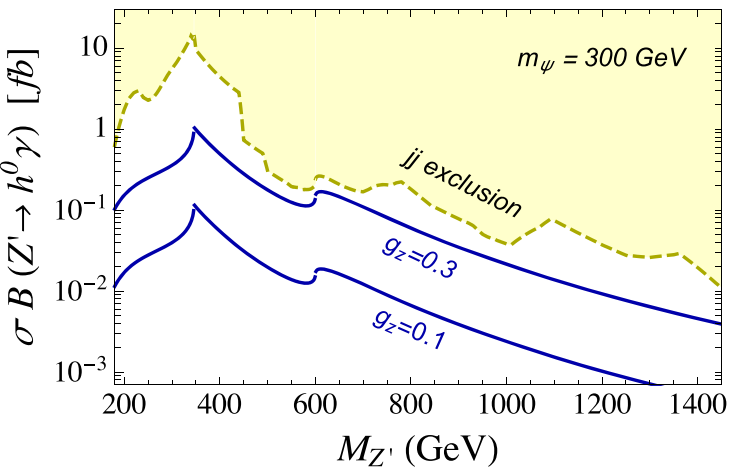

Yukawa coupling of the vectorlike leptons are fixed at $\sin \theta=0.3$ and $y_{\psi}=1$, respectively. Right panel Leading-order $p p \rightarrow Z^{\prime} \rightarrow h^{0} \gamma$ cross section at $\sqrt{s}=13 \mathrm{TeV}$ for $m_{\psi}=300 \mathrm{GeV}$ and $g_{z}=0.3$ or 0.1 . The shaded region is excluded by dijet resonance searches

permit the subdominant, loop-induced $h^{0} \gamma$ decay to be sufficiently large to be observed at the LHC. In this section we briefly discuss other models that may be explored by an $h^{0} \gamma$ search, and may represent candidates for new physics should a signal be observed in other regions of parameter space.

One challenge for the decay $Z^{\prime} \rightarrow h^{0} \gamma$ in the models discussed so far is the small branching fraction, of order $10^{-5}$ in the presence of tree-level couplings to quarks, which are necessarily present if the $Z^{\prime}$ is directly produced in protonproton collisions. An alternative is that the $Z^{\prime}$ boson is not produced directly but rather in a cascade of some heavier particle, $X_{\text {heavy }}$, for instance in association with other jets or particles that escape the detector as missing energy. In such a model, the $Z^{\prime}$ couplings to quarks may vanish at tree level, and so the decay to $h^{0} \gamma$ may have a large branching fraction. Moreover, if the additional jets are soft or the missing energy is small, the signal may still appear similar to that of a directly produced resonance. A similar scenario was considered in the context of diphoton resonances in [42]. There, it was noted that a loop-induced diboson decay width may still be small compared to a 3-body decay through an off-shell $X_{\text {heavy }}$ to jets (perhaps plus missing energy), particularly if the splitting between $X_{\text {heavy }}$ and the diboson resonance is small. A similar caveat applies here.

Another way in which new physics may give rise to a large signal in an $h^{0} \gamma$ search relative to that in dijets is to replace the photon by a pair of collimated photons arising from the decay of a highly boosted light particle. Let us construct a model of this type, which involves two complex scalars, $\phi$ and $\phi^{\prime}$, carrying the same charge $\left(z_{\phi}\right)$ under a $U(1)$ gauge symmetry. These scalars have VEVs, so that the associated gauge boson, $Z^{\prime}$, acquires a mass. In the presence of terms such as $\phi^{\dagger} \phi^{\prime} H^{\dagger} H$ in the scalar potential, the SM Higgs boson mixes with the CP-even components of $\phi$ and $\phi^{\prime}$. The CPodd components of $H, \phi$ and $\phi^{\prime}$ also mix, with two linear 
combinations becoming the longitudinal $Z$ and $Z^{\prime}$ bosons; the third one remains as a physical CP-odd scalar, $A^{0}$. As a result of mixing, there is a coupling of $A^{0}$ to the Higgs boson $h^{0}$ and the $Z^{\prime}$ :

$\frac{z_{\phi} g_{z}}{2} s_{h} Z_{\mu}^{\prime}\left(A \partial_{\mu} h+h \partial_{\mu} A\right)$,

where $g_{z}$ is the gauge coupling, and $s_{h}<1$ is a mixing parameter. This leads to a tree-level decay $Z^{\prime} \rightarrow h^{0} A^{0}$.

If $A^{0}$ couples at one loop to a pair of photons and its mass is below a few $\mathrm{GeV}$, then $A^{0}$ will be highly boosted and the two photons will appear as a single photon in the detector. Thus, the $Z^{\prime}$ may initially appear as a $h^{0} \gamma$ resonance. If the SM quarks are charged under the $U(1)$ gauge group, then the $Z^{\prime}$ gauge boson is produced at tree level at the LHC, and it can also decay into a SM quark-antiquark pair. In the case of flavor-universal quark charges, equal to $1 / 3$ as in Sect. 3.2, the ratio of the $Z^{\prime}$ widths into $h^{0} A^{0}$ and quark pairs is

$$
\frac{\Gamma\left(Z^{\prime} \rightarrow h^{0} A^{0}\right)}{\sum_{q} \Gamma\left(Z^{\prime} \rightarrow q \bar{q}\right)} \simeq \frac{3 z_{\phi}^{2} s_{h}^{2}\left(1-r_{h}^{2}\right)^{3}}{2\left(5+\left(1-r_{t}^{2}\right) \sqrt{1-4 r_{t}^{2}}\right)},
$$

where again $r_{h}=M_{h} / M_{Z^{\prime}}$ and $r_{t}=m_{t} / M_{Z^{\prime}}$. For large $M_{Z^{\prime}}$, the branching fraction is $B\left(Z^{\prime} \rightarrow h^{0} A^{0}\right) \approx s_{h}^{2} z_{\phi}^{2} / 4$. Measurements of the Higgs boson [43] currently constrain $s_{h}^{2} \lesssim 0.2$, so that $B\left(Z^{\prime} \rightarrow h^{0} A^{0}\right)$ may be as large as $5 \%$ for $z_{\phi}=1$.

The coupling of $A^{0}$ to photons is induced at one loop. The particles running in the loop may, for example, be vectorlike leptons of electric charge $Q_{L}$ and mass $m_{L}$. There is also a one-loop coupling of $A^{0}$ to gluons, induced by a top quark loop, which is suppressed by a mixing parameter, $s_{A}$, from the $\mathrm{CP}$-odd sector. The ratio of the $A^{0}$ widths into photons and gluons may be approximated for $M_{A}$ above a $\mathrm{GeV}$ by treating the gluons as massless jets:

$\frac{\Gamma\left(A^{0} \rightarrow \gamma \gamma\right)}{\Gamma\left(A^{0} \rightarrow g g\right)} \simeq \frac{\alpha^{2}}{2 \alpha_{s}^{2}}\left(Q_{L}^{2} \frac{y_{L A} m_{t}}{y_{t A} s_{A} m_{L}}+\frac{4}{3}\right)^{2}$,

where $y_{L A}$ and $y_{t A}$ are the Yukawa couplings of $A^{0}$ to the vectorlike lepton and the top quark, respectively. We neglected here the higher-order corrections in the mixing parameters. Let us choose a benchmark point in the parameter space: $Q_{L}=2, y_{L A}=y_{t A}, m_{L}=400 \mathrm{GeV}, s_{A}=0.3$. For these values, the $A^{0} \rightarrow \gamma \gamma$ branching fraction is then above $20 \%$.

When the branching fraction of $A^{0} \rightarrow \gamma \gamma$ is large, this leads to values at the percent level for an effective $B\left(Z^{\prime} \rightarrow\right.$ $h^{0 \text { “ }} \gamma$ "), where " $\gamma$ " stands for a pair of collimated photons. This is an increase by three orders of magnitude compared to the $h^{0} \gamma$ branching fraction obtained in Sect. 3, potentially turning the $h^{0} \gamma$ resonance search into a discovery channel.

The production cross section of the $Z^{\prime}$ at $\sqrt{s}=13 \mathrm{TeV}$ decreases from $100 \mathrm{pb}$ at $M_{Z^{\prime}}=300 \mathrm{GeV}$ to $60 \mathrm{fb}$ at $M_{Z^{\prime}}=2$ $\mathrm{TeV}$, for $g_{z}=0.4$, which is approximately the largest gauge coupling allowed by current dijet resonance searches [30]. Thus, the cross section times branching fraction may be as large as $0.6 \mathrm{fb}$ for a mass up to $2 \mathrm{TeV}$. Using the limit projection shown in Fig. 1, we conclude that an $h^{0 \text { " }} \gamma$ " resonance search for a $2 \mathrm{TeV} Z^{\prime}$ may set a limit with $300 \mathrm{fb}^{-1}$ of data, or may lead to discovery with $3000 \mathrm{fb}^{-1}$.

In any model featuring a decay to " $\gamma$ ", the parameter space will be limited by the requirement that the light state $A^{0}$ decays to two photons before reaching the electromagnetic calorimeter and is indeed reconstructed as a single photon [912]. Although the diphoton decay can have a sizable branching fraction, the lifetime is dominated, for masses above a $\mathrm{GeV}$, by the width into gluons,

$\Gamma\left(A^{0} \rightarrow g g\right) \simeq \frac{\alpha_{s}^{2} y_{t A}^{2} s_{A}^{2}}{64 \pi^{3} m_{t}^{2}} M_{A}^{3}$.

The typical separation between the photons produced in the decay of a state with a total width $\Gamma_{A}$ and boost $\gamma_{A}$, at distance $R$ from its production, is

$\Delta \theta_{\gamma \gamma} \approx\left(1-\frac{\gamma_{A} \beta_{A}}{R \Gamma_{A}}\right) \frac{2}{\gamma_{A}}$.

In order that the collimated photons are reconstructed as a single photon, this opening angle cannot be too large. For instance, the innermost calorimeter layer at ATLAS is designed to reject pions and has segmentation $\Delta \eta_{\text {ATLAS }}=$ $3 \times 10^{-3}$, placing an upper bound on $M_{A}$ that grows with the $Z^{\prime}$ mass (due to the $A^{0}$ boost). Meanwhile, the $A^{0}$ must decay on average before the inner layer of the calorimeter, a distance of $O(1.3) \mathrm{m}$, which places a lower bound on $M_{A}$. While it is possible to simultaneously satisfy these constraints, a full analysis should take into account the temporal and angular distributions of $A^{0}$ decays.

So, for certain choices of $M_{A}$ and $M_{Z^{\prime}}$, it may be possible that a resonance be observed in an $h^{0} \gamma$ search with the "photon" comprised of collimated photons. Subsequent studies may be able to use photon conversions or shower profile to distinguish between a single photon and boosted diphoton [23,44-46]. In particular, these studies may be particularly valuable if an observation is made without either a corresponding observation in dijets or dileptons, potentially suggesting these channels are forbidden or suppressed relative to the values expected in the models discussed in Sect. 3 .

We have been discussing a new vector decaying into $h^{0 \text { " }} \gamma$ " but the resonance could also be a (pseudo)scalar. A scalar cannot decay directly to $h^{0} \gamma$, but again it may appear in this channel if it decays to a pair of collimated photons, e.g., $A^{\prime} \rightarrow$ $A h^{0}$. Such a decay can occur via trilinear terms in the scalar potential. Moreover, if the $A^{\prime}$ were produced via gluon fusion through a loop of new heavy, colored particles, the tree-level decay to a Higgs and a " $\gamma$ " could readily dominate over the loop-level decay to dijets. In this case, an $h^{0} \gamma$ search may 
even represent the most promising approach for discovering the new resonance.

\section{Conclusions}

The LHC experiments have carried out searches for many diboson resonances, with a notable exception: a search for a resonance consisting of the SM Higgs boson and a photon. We have discussed simple models containing a vector resonance, $Z^{\prime}$, which decays at one loop to this $h^{0} \gamma$ final state. The $Z^{\prime}$ is produced from its coupling to light quarks so the branching fraction to $h^{0} \gamma$ is of the order of $\sim 10^{-5}$. Typically one would also expect the $Z^{\prime}$ to decay to lepton pairs at a comparable rate (though this need not be the case), and to a pair of jets nearly $100 \%$ of the time. Despite the small branching fraction, there are viable models with $p p \rightarrow Z^{\prime} \rightarrow h^{0} \gamma$ cross section larger than $1 \mathrm{fb}$, for $M_{Z^{\prime}}$ in the $200-450 \mathrm{GeV}$ range.

Larger branching fractions to the $h^{0} \gamma$ final state can be achieved if the photon is not a single photon but rather a collimated pair of photons produced in the decay of a light pseudoscalar, $Z^{\prime} \rightarrow h A^{0} \rightarrow h^{\prime \prime} \gamma$ ”. For resonance mass above $\sim 1 \mathrm{TeV}$ the decay products of the Higgs boson would be boosted and, for the dominant $b \bar{b}$ mode, would be reconstructed as a single jet with substructure. Excited quark searches look for a jet+photon final state and we recast the existing searches to estimate the reach of a $q^{*}$ search, augmented by a Higgs tagger applied to the jet. We estimated it is possible to discover a $Z^{\prime}$ using this technique up to masses of about $2 \mathrm{TeV}$, and we presented a model where the branching fraction to $h^{0 \text { " }} \gamma$ " is relatively large, at the percent level.

Although the decay of a spin- 0 particle into $h^{0} \gamma$ is forbidden, a heavy neutral scalar may cascade decay into the Higgs boson and collimated photons, leading again to an $h^{0}$ " $\gamma$ " final state. The production cross section of a scalar from gluon fusion is smaller than that of a $Z^{\prime}$ coupled at tree level to first-generation quarks, so the mass range accessible for discovery in this case is reduced. If a Higgs-photon resonance is discovered, the angular distribution may be needed to identify the spin of the heavy particle.

In addition to resonances decaying to a SM Higgs and a photon it is possible that there are other Higgs-like scalars, $h^{\prime}$, which could be produced in the decay $Z^{\prime} \rightarrow h^{\prime} \gamma$. For $h^{\prime}$ mass below the $W^{+} W^{-}$threshold the final states are the same as for $h^{0} \gamma$ but the kinematics are different. Dedicated analyses for $h^{0} \gamma$ and $h^{\prime} \gamma$, at both light and heavy resonance masses, could uncover a new boson coupled to quarks (a $Z^{\prime}$, or even a scalar in the case of collimated photons), or allow for further characterization of a resonance found through other final states, e.g., lepton or jet pairs. We strongly advocate for the inclusion of these final states in the diboson search program.

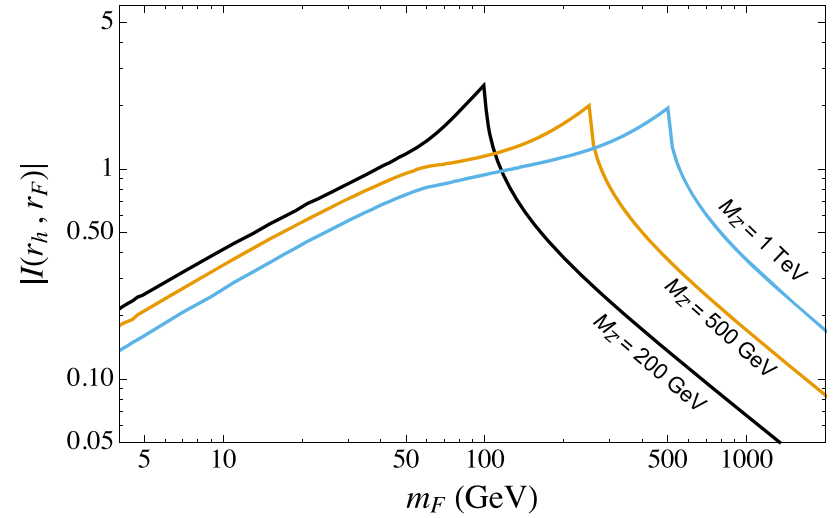

Fig. 5 The loop integral $\left|I\left(M_{h} / M_{Z^{\prime}}, m_{F} / M_{Z^{\prime}}\right)\right|$ of Eq. (A.2) as a function of the fermion mass, for three values of $M_{Z^{\prime}}$

Acknowledgements This work was supported by the DoE under Contract Number DE-SC0007859 and Fermilab, operated by Fermi Research Alliance, LLC under Contract Number DE-AC02-07CH11359 with the United States Department of Energy.

Open Access This article is distributed under the terms of the Creative Commons Attribution 4.0 International License (http://creativecomm ons.org/licenses/by/4.0/), which permits unrestricted use, distribution, and reproduction in any medium, provided you give appropriate credit to the original author(s) and the source, provide a link to the Creative Commons license, and indicate if changes were made.

Funded by $\mathrm{SCOAP}^{3}$.

\section{A Appendix: The loop integral}

The contributions of a fermion loop (see Fig. 2) to the $Z^{\prime} \rightarrow$ $h^{0} \gamma$ width depend on a function $f$ introduced in Eq. (3.12). This is given by

$f\left(r_{h}^{2}, r_{F}^{2}\right)=\left(1-r_{h}^{2}\right)^{3}\left|I\left(r_{h}, r_{F}\right)\right|^{2}$,

where the first term is a phase-space factor, and the second term arises from the loop integral.

The two diagrams shown in Fig. 2 yield equal CPconserving contributions, giving a factor of 2 in the amplitude. After introducing Feynman parameters $x$ and $y$ and computing the loop momentum integrals, we find that the function $I\left(r_{h}, r_{F}\right)$ that enters the $Z^{\prime} \rightarrow h^{0} \gamma$ amplitude is given by

$$
\begin{aligned}
I\left(r_{h}, r_{F}\right)= & r_{F} \int_{0}^{1} \mathrm{~d} x \int_{0}^{1-x} \mathrm{~d} y \frac{4 x y-1}{\left(1-r_{h}^{2}\right) x y-x(1-x)+r_{F}^{2}-i \epsilon} \\
= & \frac{r_{F}}{1-r_{h}^{2}}\left[2+\int_{0}^{1} \frac{\mathrm{d} x}{x}\left(4 \frac{x(1-x)-r_{F}^{2}}{1-r_{h}^{2}}-1\right)\right. \\
& \left.\times \log \left(\frac{r_{h}^{2} x(1-x)-r_{F}^{2}+i \epsilon}{x(1-x)-r_{F}^{2}+i \epsilon}\right)\right]
\end{aligned}
$$

where $\epsilon$ is the imaginary part of the Feynman propagator for the fermion running in the loop, rescaled by a mass- 
dependent factor. In the case of an unstable fermion of total width $\Gamma_{F}, \epsilon$ is a physical parameter: $\epsilon=m_{F} \Gamma_{F} / M_{Z^{\prime}}^{2}$. For a stable fermion $\epsilon \rightarrow 0_{+}$is the usual Feynman propagator prescription. While the remaining $x$-integral can be done analytically the result is not illuminating. Instead, we compute the integral numerically as a function of $m_{F}$ and plot it in Fig. 5 for a few choices of $M_{Z^{\prime}}$. We also compute the integral analytically in two limits:

$$
\begin{aligned}
& I\left(r_{h}, r_{F}\right) \\
& \quad= \begin{cases}\frac{2 r_{F}}{1-r_{h}^{2}}\left[1+\left(\frac{2}{1-r_{h}^{2}}+\log \frac{r_{F}^{2}}{r_{h}}\right) \log r_{h}\right] & \text { if } r_{F} \ll r_{h} . \\
-\frac{1}{3 r_{F}} & \text { if } r_{F} \gg 1,\end{cases}
\end{aligned}
$$

where we took into account that $r_{h}<1$.

\section{References}

1. N. Craig, P. Draper, K. Kong, Y. Ng, D. Whiteson, The unexplored landscape of two-body resonances. arXiv:1610.09392 [hep-ph]

2. G. Aad et al. [ATLAS Collaboration], Search for a new resonance decaying to a $\mathrm{W}$ or Z boson and a Higgs boson in the $\ell \ell / \ell v / v v+b \bar{b}$ final states. Eur. Phys. J. C 75(6), 263 (2015). arXiv:1503.08089

3. M. Aaboud et al. [ATLAS Collaboration], Search for new resonances decaying to a $W$ or $Z$ boson and a Higgs boson in the $\ell^{+} \ell^{-} b \bar{b}, \ell v b \bar{b}$, and $v \bar{v} b \bar{b}$ channels with $p p$ collisions at $\sqrt{s}=13$ TeV. Phys. Lett. B 765, 32 (2017). arXiv:1607.05621

4. M. Aaboud et al. [ATLAS Collaboration], Search for heavy resonances decaying to a $\mathrm{W}$ or Z boson and a Higgs boson in the $q \bar{q}^{(\prime)} b \bar{b}$ final state in $p p$ collisions at $\sqrt{s}=13 \mathrm{TeV}$. Report CONF-2017-018 (2017)

5. V. Khachatryan et al. [CMS Collaboration], Search for massive WH resonances decaying into the $\ell \nu \mathrm{b} \overline{\mathrm{b}}$ final state at $\sqrt{s}=8 \mathrm{TeV}$. Eur. Phys. J. C 76(5), 237 (2016). arXiv:1601.06431

6. V. Khachatryan et al. [CMS Collaboration], Search for a massive resonance decaying into a Higgs boson and a $\mathrm{W}$ or $\mathrm{Z}$ boson in hadronic final states in proton-proton collisions at $\sqrt{s}=8 \mathrm{TeV}$. JHEP 1602, 145 (2016). arXiv: 1506.01443

7. V. Khachatryan et al. [CMS Collaboration], Search for heavy resonances decaying into a vector boson and a Higgs boson in final states with charged leptons, neutrinos, and b quarks. Phys. Lett. B 768, 137 (2017). arXiv: 1610.08066

8. V. Khachatryan et al. [CMS Collaboration], Search for heavy resonances decaying into a vector boson and a Higgs boson in hadronic final states with 2016 data. Report CMS-PAS-B2G-17-002 (2017)

9. B.A. Dobrescu, G.L. Landsberg, K.T. Matchev, Higgs boson decays to CP odd scalars at the Tevatron and beyond. Phys. Rev. D 63, 075003 (2001). arXiv:hep-ph/0005308

10. N. Toro, I. Yavin, Multiphotons and photon jets from new heavy vector bosons. Phys. Rev. D 86, 055005 (2012). arXiv:1202.6377

11. P. Draper, D. McKeen, Diphotons from tetraphotons in the decay of a 125 GeV Higgs at the LHC. Phys. Rev. D 85, 115023 (2012). arXiv: 1204.1061

12. S.D. Ellis, T.S. Roy, J. Scholtz, Jets and photons. Phys. Rev. Lett. 110, 122003 (2013). arXiv: 1210.1855

13. J. Alwall, R. Frederix, S. Frixione, V. Hirschi, F. Maltoni, O. Mattelaer et al., The automated computation of tree-level and nextto-leading order differential cross sections, and their matching to parton shower simulations. JHEP 07, 079 (2014). doi:10.1007/ JHEP07(2014)079. arXiv:1405.0301

14. H. Khanpour, S. Khatibi, M. Mohammadi Najafabadi, Probing Higgs boson couplings in $\mathrm{H}+\gamma$ production at the LHC. arXiv:1702.05753 [hep-ph]

15. V. Khachatryan et al. [CMS collaboration], Search for excited quarks in the $\gamma+$ jet final state in proton-proton collisions at $\sqrt{s}=8$ TeV. Phys. Lett. B 738, 274 (2014). doi:10.1016/j.physletb.2014. 09.048. arXiv: 1406.5171

16. V. Khachatryan et al. [CMS collaboration], Search for excited quarks in the photon+jet final state in proton-proton collisions at 13 TeV. Report PAS-EXO-16-015 (2016)

17. G. Aad et al. [ATLAS collaboration], Search for new phenomena in photon+jet events collected in proton-proton collisions at $\sqrt{s}=8$ TeV, Phys. Lett. B 728, 562 (2014). doi:10.1016/j.physletb.2013. 12.029. arXiv: 1309.3230

18. G. Aad et al. [ATLAS collaboration], Search for new phenomena with photon+jet events in proton-proton collisions at $\sqrt{\mathrm{s}}=$ 13 TeV. JHEP 03, 041 (2016). doi:10.1007/JHEP03(2016)041. arXiv: 1512.05910

19. G. Aad et al. [CMS collaboration], Search for excited states of light and heavy flavor quarks in the $\gamma+$ jet final state in proton-proton collisions at $\sqrt{s}=13 \mathrm{TeV}$. Report CMS-PAS-EXO-17-002

20. T. Sjostrand, S. Mrenna, P.Z. Skands, PYTHIA 6.4 physics and manual. JHEP 05, 026 (2006). doi:10.1088/1126-6708/2006/05/ 026. arXiv:hep-ph/0603175

21. J. de Favereau, C. Delaere, P. Demin, A. Giammanco, V. Lematre, A. Mertens et al., DELPHES 3, A modular framework for fast simulation of a generic collider experiment. JHEP 02, 057 (2014). doi:10.1007/JHEP02(2014)057. arXiv:1307.6346

22. M. Cacciari, G.P. Salam, G. Soyez, FastJet user manual. Eur. Phys. J. C 72, 1896 (2012). doi:10.1140/epjc/s10052-012-1896-2. arXiv: 1111.6097

23. G. Aad et al. [ATLAS collaboration], Search for new phenomena in events with at least three photons collected in $p p$ collisions at $\sqrt{s}=8$ TeV. Eur. Phys. J. C 76, 210 (2016). doi:10.1140/epjc/ s10052-016-4034-8. arXiv:1509.05051

24. V. Khachatryan et al. [CMS collaboration], Search for resonances and quantum black holes using dijet mass spectra in proton-proton collisions at $\sqrt{s}=8$ TeV. Phys. Rev. D 91, 052009 (2015). doi: 10. 1103/PhysRevD.91.052009. arXiv:1501.04198

25. G. Aad et al. [ATLAS Collaboration], Search for high-mass dilepton resonances in pp collisions at $\sqrt{s}=8 \mathrm{TeV}$. Phys. Rev. D 90, 052005 (2014). doi:10.1103/PhysRevD.90.052005. arXiv: 1405.4123

26. M. Carena, A. Daleo, B.A. Dobrescu, T.M.P. Tait, $Z^{\prime}$ gauge bosons at the Tevatron. Phys. Rev. D 70, 093009 (2004). arXiv:hep-ph/0408098

27. P. Fileviez Perez, M.B. Wise, Breaking local baryon and lepton number at the TeV Scale. JHEP 1108, 068 (2011). arXiv: 1106.0343

28. M. Duerr, P. Fileviez Perez, M.B. Wise, Gauge theory for baryon and lepton numbers with leptoquarks. Phys. Rev. Lett. 110, 231801 (2013). arXiv:1304.0576

29. M. Duerr, P. Fileviez, Perez, Baryonic dark matter. Phys. Lett. B 732, 101 (2014). arXiv:1309.3970

30. B.A. Dobrescu, F. Yu, Coupling-mass mapping of dijet peak searches. Phys. Rev. D 88(3), 035021 (2013). Erratum: [Phys. Rev. D 90(7), 079901 (2014)]. arXiv:1306.2629

31. B.A. Dobrescu, C. Frugiuele, Hidden GeV-scale interactions of quarks. Phys. Rev. Lett. 113, 061801 (2014). arXiv:1404.3947

32. P. Fileviez Perez, S. Ohmer, H.H. Patel, Minimal theory for LeptoBaryons. Phys. Lett. B 735, 283 (2014). arXiv:1403.8029

33. A. Alloul, N.D. Christensen, C. Degrande, C. Duhr, B. Fuks, FeynRules 2.0: a complete toolbox for tree-level phenomenology. Comput. Phys. Commun. 185, 2250 (2014). arXiv:1310.1921 
34. CMS Collaboration, Searches for dijet resonances in pp collisions using data collected in 2016. Report PAS-EXO-16-056(2017)

35. CMS Collaboration, Search for light vector resonances decaying to quarks at $\sqrt{s}=13 \mathrm{TeV}$. Report EXO-16-030 (2016)

36. V. Khachatryan et al. [CMS Collaboration], Search for narrow resonances in dijet final states at $\sqrt{s}=8 \mathrm{TeV}$ with the novel CMS technique of data scouting. Phys. Rev. Lett. 117(3), 031802 (2016). arXiv: 1604.08907

37. ATLAS Collaboration, Search for light dijet resonances with the ATLAS detector using a Trigger-Level Analysis in LHC pp collisions at $\sqrt{s}=13 \mathrm{TeV}$. Report CONF-2016-030 (2016)

38. ATLAS Collaboration, Search for new light resonances decaying to jet pairs and produced in association with a photon or a jet in proton-proton collisions at $\sqrt{s}=13 \mathrm{TeV}$. Report CONF-2016-070 (2016)

39. G. Aad et al. [ATLAS Collaboration], Search for new phenomena in the dijet mass distribution using $p-p$ collision data at $\sqrt{s}=8$ TeV. Phys. Rev. D 91(5), 052007 (2015). arXiv:1407.1376

40. T. Aaltonen et al. [CDF Collaboration], Search for new particles decaying into dijets in proton-antiproton collisions at $\sqrt{s}=1.96$ TeV. Phys. Rev. D 79, 112002 (2009). arXiv:0812.4036
41. A. Falkowski, D.M. Straub, A. Vicente, Vector-like leptons: Higgs decays and collider phenomenology. JHEP 1405, 092 (2014). arXiv: 1312.5329

42. B.A. Dobrescu, P.J. Fox, J. Kearney, J. Phys. G 44(6), 065003 (2017). arXiv: 1605.08772

43. G. Aad et al. [ATLAS and CMS Collaborations], Measurements of the Higgs boson production and decay rates and constraints on its couplings from a combined ATLAS and CMS analysis of the LHC pp collision data at $\sqrt{s}=7$ and 8 TeV. JHEP 1608, 045 (2016). arXiv: 1606.02266

44. P. Agrawal, J. Fan, B. Heidenreich, M. Reece, M. Strassler, Experimental considerations motivated by the diphoton excess at the LHC. JHEP 1606, 082 (2016). arXiv: 1512.05775

45. S. Knapen, T. Melia, M. Papucci, K. Zurek, Rays of light from the LHC. Phys. Rev. D 93, 075020 (2016). doi:10.1103/PhysRevD.93. 075020. arXiv: 1512.04928

46. B. Dasgupta, J. Kopp, P. Schwaller, Photons, photon jets, and dark photons at $750 \mathrm{GeV}$ and beyond. Eur. Phys. J. C 76, 277 (2016). doi:10.1140/epjc/s10052-016-4127-4. arXiv:1602.04692 